Ann. Biol. anim. Bioch. Biophys., I970, $10(4), 643-657$.

\title{
TAILLE ET STRUCTURE DES PARTIGULES VÉGÉTALES AU NIVEAU DU FEUILLET ET DES F ĖCES CHEZ LES BOVINS
}

\author{
Élisabeth GRENET \\ Station de Recherches sur l'élevage des Ruminants, \\ Centre de Recherches de Clermont-Ferrand, 63-Saint-Genès-Champanelle \\ Institut national de la Recherche agronomique
}

RÉSUMÉ

Nous avons étudié la taille des particules du contenu de feuillet et des fèces, la composition microscopique des particules vćgétales et la proportion de tissus lignifiés qu'elles renferment, sur 50 échantillons de contenu de feuillet et 48 échantillons de fèces de bovins. 20 régimes, différents ont été étudiés dont 6 étaient des régimes de fourrages verts et I4 des régimes d'engraissement hivernal à base de fourrages et d'aliment concentré. Nous avons relié nos résultats avec la composition chimique et la digestibilité du fourrage ingéré.

La taille moyenne des particules du contenu de feuillet est très faible, $0,50 \mathrm{~mm}$ en moyenne pour les 20 régimes étudiés, et la taille moyenne des particules fécales est encore inférieure $(0,39 \mathrm{~mm})$. La taille moyenne des particules du feuillet varie en sens inverse de la digestibilité de la matière organique des fourrages, et dans le même sens que la teneur en matière organique indigestible et la teneur en cellulose brute indigestible. La taille moyenne des particules fécales montre seulement une relation positive avec la teneur en matière organique indigestible.

Les particules du contenu de feuillet et des fèces de taille supérieure à o,40 mm sont composées de fragments de tissus lignifiés (vaisseaux et sclérenchyme), de parenchyme et d'épiderme.

L'examen microscopique des contenus de feuillet et des fèces fermet d'identifier en grande partie les divers constituants du régime, betteraves et aliments concentrés, ainsi que le lieu de la digestion de l'amidon des céréales.

La lignification des particules du contenu de feuillet montre une relation positive avec la lignification des particules fécales. Elles présentent toutes les deux une liaison négative avec la digestibilité de la matière organique et de la cellulose brute des fourrages, et une liaison directe avec la teneur en matière organique indigestible et la tencur en cellulose brute indigestible.

\section{INTRODUCTION}

Nous avons montré, au cours d'une étude antérieure, que la taille moyenne des particules fécales des moutons est faible, inférieure au millimètre (GRENET, Ig66). L'examen microscopique des plus grosses particules, de taille supérieure à $0,40 \mathrm{~mm}$, 
avait montré qu'elles sont constituées de tissu lignifié, de lambeaux d'épiderme et de parenchyme; la taille moyenne des particules fécales ainsi que leur lignification varient en sens inverse de la digestibilité de la matière organique et de la teneur en cellulose brute des fourrages. L'importance de la digestion des particules au cours du transit intestinal peut être mise en évidence par l'étude microscopique comparative des particules des contenus de feuillet, qui ont subi la digestion microbienne dans le rumen, et des particules fécales. De plus, l'emploi de méthodes microscopiques pour l'étude de la digestion peut faciliter l'interprétation des résultats obtenus par les méthodes chimiques.

Très peu d'études ont été effectuées sur ce sujet. BECKER $e t$ al. (Ig63) ont mesuré la taille des particules des différents réservoirs gastriques du veau, et ont montré que 68 p. Ioo des particules du contenu de feuillet étaient de taille inférieure au $\mathrm{mm}$. SMrTh et al. ( $\mathrm{r} 967$ ) aux États-Unis, après avoir mis en évidence l'importance des très fines particules $(<74 \mu)$ dans les fèces de mouton (Io p. Ioo des membranes contenues dans les fèces), ont étudié la taille des particules qui transitent hors du rumen ; ceci, à l'aide d'administration par la fistule du rumen d'un mouton, de blé plante entière dont les membranes étaient marquées au carbone $\mathrm{r}_{4}$. Une canule duodénale permettait de vérifier la taille des particules sortant du rumen. D'après ces auteurs il n'existe qu'une faible probabilité pour que les particules de taille supérieure à la maille du tamis de $840 \mu$ quittent le rumen; elles sont d'abord réduites à l'état de plus fines particules. PEARCE (I967), en Australie, a mesuré la taille des particules alimentaires et celle des particules du rumen et des fèces de mouton, ces dernières étant les plus petites. Dans les conditions particulières d'un seul repas par jour, il a mis en évidence la diminution de la quantité de grosses particules dans le rumen jusqu'à 24 hetures après le repas, tandis que le poids des petites particules augmente jusqu'à 4 heures après le repas avant de diminuer.

Trotisen et Campbeil (I968), au Canada, ont étudié la taille des particules des différents réservoirs gastriques de moutons abattus environ 3 heures après le repas, alimentés avec des foins de nature et d'âge différents, ingérés en quantités variables, et ont fait apparaittre des variations de la forme des particules suivant l'espèce fourragère considérée.

Nous avons mesuré la taille des particules du contenu de feuillet et des fèces de bovins alimentés avec 20 régimes très différents, soit de fourrages verts, soit de foins et d'aliments concentrés, et nous avons étudié la structure microscopioue de ces particules, ce qui, à notre connaissance, n'a jamais été effectué.

\section{MATÉRIEL E'T MÉTHODES}

\section{Choix et constitution des échantillons}

Nous avons prélevé nos échantillons sur 50 taurillons, bøufs et vaches alimentés avec 20 régimes différents (tabl. I), dans le cadre d'expériences sur la production de viande, réalisées à la Station par $\mathrm{C}$. BÉranger. Parmi ces 20 régimes, 6 sont des régimes de fourrages verts dont les 4 premiers ont été apportés à l'auge et les 2 autres pâturés, et $\mathrm{I} 4$ sont des régimes d'engraissement hivernal à base de fourrages et d'aliment concentré en proportions variables.

Les essais no 2,3 et 4 visent à comparer différentes formes de présentation d'un même fourrage : luzerne présentée sous forme de foin long, de foin condensé, et de produit déshydraté et 
condensé (aggloméré après broyage) (essai 2), foin de luzerne présenté sous forme longue et condensée (essai 3 ), foin de graminées présenté sous forme longue et condensée (essai 4 ). Le régime no 5 est composé essentiellement de luzerne déshydratée condensée, consommée par des vaches de réforme. Dans l'essai $n^{\circ} 6$, des taurillons ont reçu du foin de luzerne et du maîs sous forme de mouture grossière, soit cru (maïs normal), soit ayant subi un traitement industriel de trempage dans l'eau puis séchage sur rouleaux à une température de $60-70^{\circ} \mathrm{C}$ (maîs traité). Les régimes $n^{\circ} 7^{a}$ et $7^{b}$ comprennent un même foin de trèfle distribué avec un aliment concentré à des vaches de réforme dans des proportions différentes. Le régime no 8 est à base de foin de pré et d'ensilage d'herbe, et le régime no 9 est composé en majeure partie d'orge reconstituée (paille et grains mélangés en proportions identiques) condensée. La composition de ces régimes, les quantités journalières consommées par les animaux (excepté pour les animaux au pâturage) et les coefficients de digestibilité de la matière organique et de la cellulose brute Weende sont donnés dans le tableau $\mathrm{I}$. Ces coefficients ont été mesurés sur des moutons, sauf pour le régime no 9 pour lequel des bœufs ont été utilisés.

Nous avons prélevé chaque jour à la même heure, lors du changement de la litière des animaux, un échantillon de $\mathrm{I} \mathrm{kg}$ environ de fèces par animal, durant les 5 jours précédant l'abattage. Pour les deux régimes d'herbe pâturée $(\mathrm{x} e, f)$ un échantillon global de $\mathbf{r} \mathrm{kg}$ environ de fèces était prélevé chaque jour sur l'ensemble des bouses fraîches pendant les 5 jours précédant l'abattage. Ces échantillons étant conservés au froid, un échantillon moyen pondéré de $500 \mathrm{~g}$ a été constitué par animal pour les animaux en stabulation, et par régime pour les animaux au pâturage.

Les animaux ont été abattus 24 heures environ après leur dernier repas, excepté ceux qui étaient au pâturage et dont l'abattage a eu lieu 8 heures environ après leur départ du pâturage. Dès l'abattage, le tractus digestif a été sorti de la cavité abdominale et le feuillet séparé par section, la ferme consistance de son contenu ainsi que sa situation entre les lames empêchant tout mélange avec le contenu des autres compartiments. Le feuillet a été vidé complètement et un échantillon de $\mathrm{I} \mathrm{kg}$ environ de contenu prélevé.

Tous les échantillons ont été conservés à - $5^{\circ} \mathrm{C}$, excepté les fèces correspondant aux régimes $\mathrm{I} a, b, c$, qui ont été séchées à l'étuve à $80^{\circ} \mathrm{C}$ pendant 48 heures.

\section{Séparation des particules des contenus de feuillet et des fèces selon leur taille}

La mesure de la taille des particules a été effectuée sur les échantillons de chaque animal. La teneur en matière sèche des échantillons est déterminée en double sur Io g de produit. D'autre part, deux prises de ro g de matière fraîche sont mises à tremper dans un cristallisoir avec environ $60 \mathrm{ml}$ d'eau pendant au moins 24 heures à la température ambiante. Elles sont ensuite tamisées en milieu liquide, sur 4 tamis à maille d'ouverture respective I $\mathrm{mm}, 0,40 \mathrm{~mm}, 0, \mathrm{I} 6 \mathrm{~mm}$ et o, Io $\mathrm{mm}$, à l'aide d'une tamiseuse électro-magnétique Frirsch: équipée d'un couvercle avec tête à douche et d'un bac de récupération. La méthode utilisée pour séparer et récupérer les particules a été précédemment exposée (GRENET, I966).

Une quantité variable de l'échantillon ( $\mathrm{x}$ à $20 \mathrm{p}$. Ioo de la matière sèche totale), est perdue au cours de l'analyse granulométrique, ainsi que nous l'avions observé lors de l'étude des particules végétales des fèces de mouton; il s'agit de produits solubles dans l'eau et de très fines particules non retenues par les filtres.

Le calcul de la taille moyenne des particules est effectué en portant en ordonnée le pourcentage cumulé de particules traversant les mailles de chaque tamis, et en abscisse le diamètre des mailles de ces tamis (fig. I). L'observation nous a montré que la majorité des particules recueillies sur le tamis à maille de $\mathrm{I} \mathrm{mm}$ ont une taille inférieure à $2,5 \mathrm{~mm}$. C'est pourquoi nous avons considéré cette taille comme maximum. A partir de la courbe ainsi obtenue, nous avons déterminé pour des pourcentages allant de 5 à $95 \mathrm{p}$. Ioo, tous les $5 \mathrm{p}$. roo, la taille théorique des particules correspondante. La moyenne arithmétique des iq valeurs obtenues exprime la taille moyenne des particules. Ce mode de calcul diffère de celui que nous avions utilisé précédemment (GRENET, I966) : nous avions, en effet, calculé une taille moyenne pondérée en considérant que la taille des particules retenues par un tamis était égale au diamètre des mailles de ce tamis, ce qui était une estimation par défaut de la taille réelle.

\section{Examen microscopique des particules}

L'examen microscopique des particules a été effectué sur un échantillon moyen des particules provenant des 2 ou 3 animaux qui ont reçu le même régime. Les particules recueillies sur les tamis $\mathrm{I}$ et $0,4^{\circ} \mathrm{mm}$ sont soumises séparément à une double coloration et une note est attribuée à chaque particule, variant de o à ro suivant la proportion de tissus lignifiés par rapport 


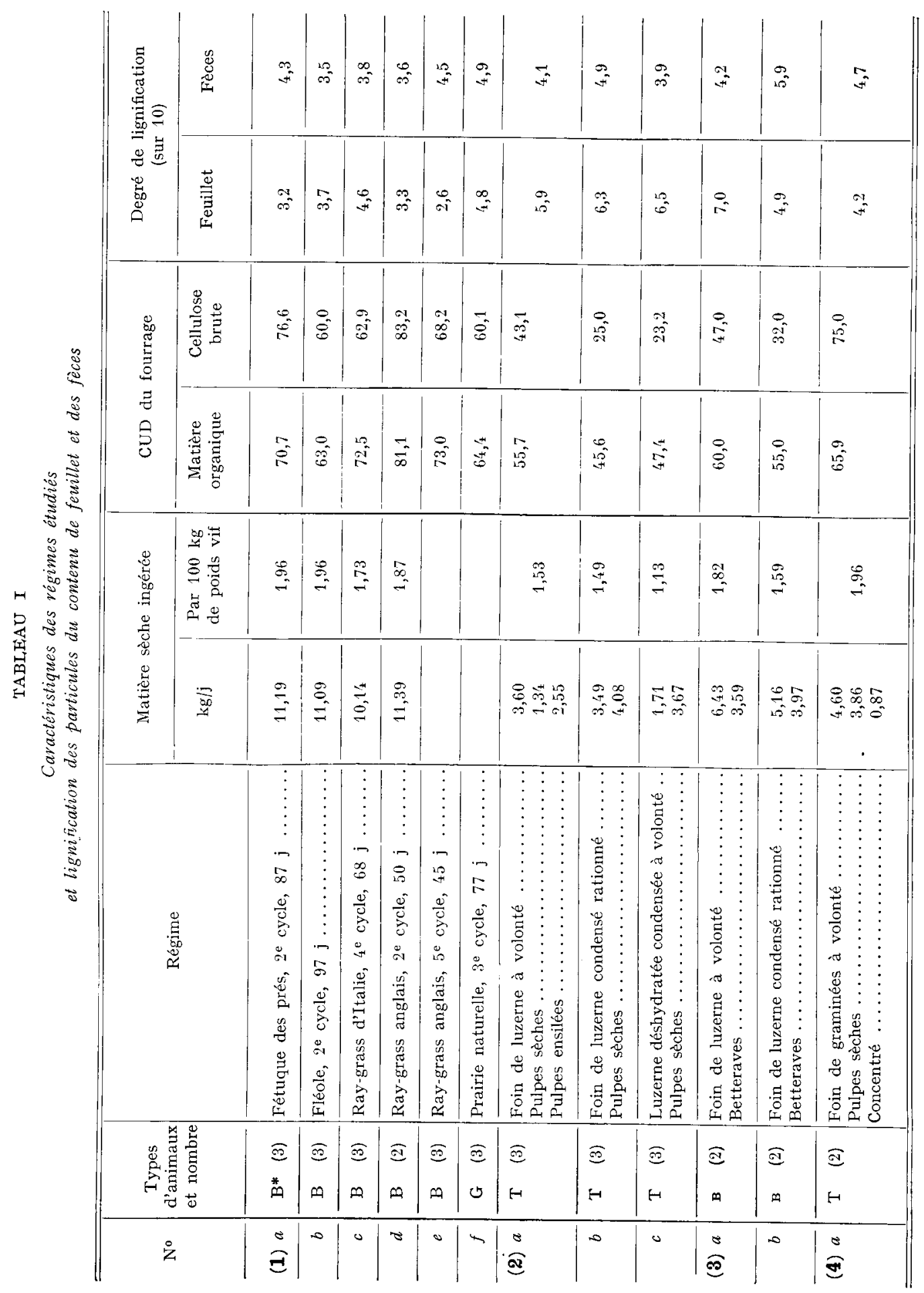


PARTICULES DU FEUILLET ET DES FÈCES

\begin{tabular}{|c|c|c|c|c|c|c|c|c|c|}
\hline 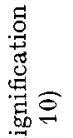 & $\begin{array}{l}\text { 总 } \\
\text { 出 }\end{array}$ & $\overline{i s}$ & is & is & 15 & $\underbrace{20}_{00}$ & 9 & $\hat{i s}$ & $\begin{array}{l}0 \\
10^{\circ}\end{array}$ \\
\hline 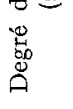 & 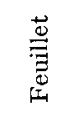 & $\therefore$ & 13 & is & $\overrightarrow{0}$ & $g$ & जी & 3 & 8 \\
\hline \begin{tabular}{l} 
品 \\
\multirow{\Xi}{5}{} \\
$\bar{\Xi}$
\end{tabular} & 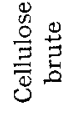 & & 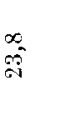 & ") & & 今8 & & 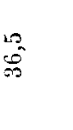 & 命 \\
\hline 点 & 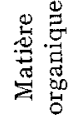 & & $\overrightarrow{5}$ & $\overline{\hat{i}}$ & & 势 & & 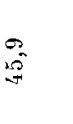 & 8 \\
\hline 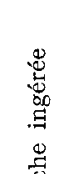 & 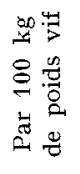 & $\stackrel{\substack{\infty \\
-}}{-}$ & 今i & $\underset{-}{\stackrel{\infty}{-}}$ & 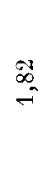 & $\begin{array}{l}\overrightarrow{10} \\
\vec{i}\end{array}$ & 然 & 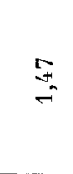 & $\stackrel{\mathscr{g}}{\rightarrow}$ \\
\hline
\end{tabular}

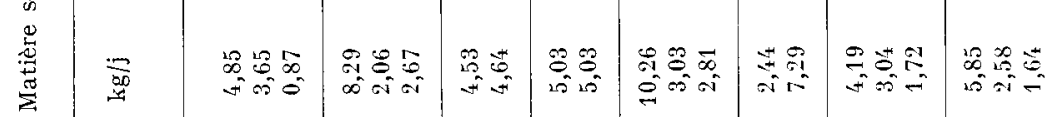

\begin{tabular}{|c|c|c|c|c|c|c|c|c|}
\hline 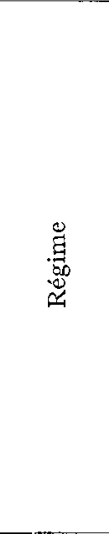 & 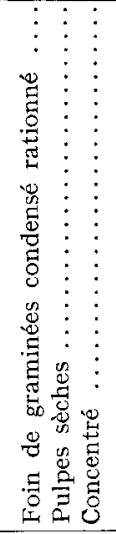 & 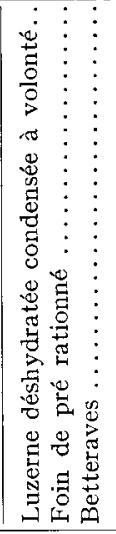 & 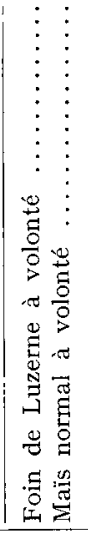 & 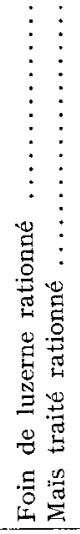 & 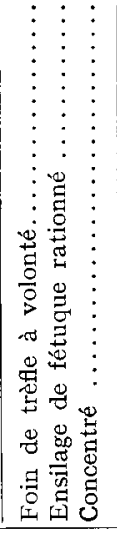 & 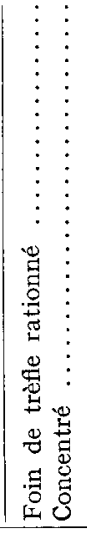 & 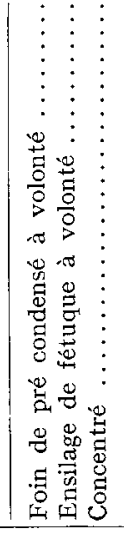 & 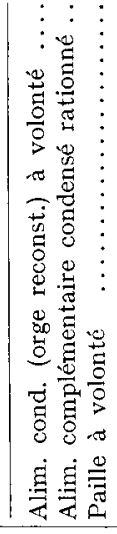 \\
\hline 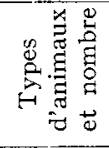 & $\begin{array}{l}\widehat{\Theta} \\
\leftrightarrow\end{array}$ & $\begin{array}{l}\widehat{\mathfrak{O}} \\
>\end{array}$ & $\begin{array}{l}\text { बㅁ } \\
-\end{array}$ & $\begin{array}{l}\widehat{3} \\
H\end{array}$ & $\begin{array}{l}\widehat{I} \\
>\end{array}$ & $\begin{array}{l}\overline{9} \\
>\end{array}$ & $\begin{array}{l}\widehat{\mathbb{N}} \\
\infty\end{array}$ & $\begin{array}{l}\widehat{\text { S }} \\
H\end{array}$ \\
\hline$\stackrel{\circ}{2}$ & $\therefore$ & $\sqrt{3}$ & $\stackrel{\oplus}{\oplus}$ & $\infty$ & $\stackrel{\circ}{E}$ & $\circ$ & $\widehat{\infty}$ & $\widehat{\Phi}$ \\
\hline
\end{tabular}

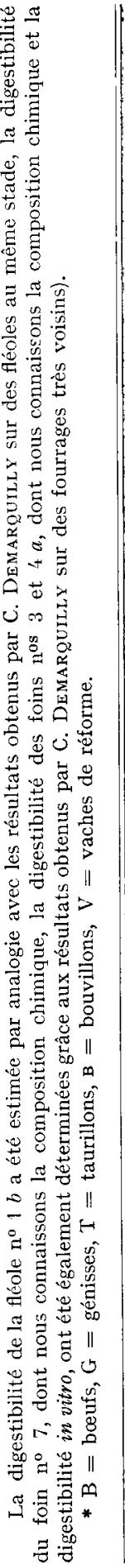



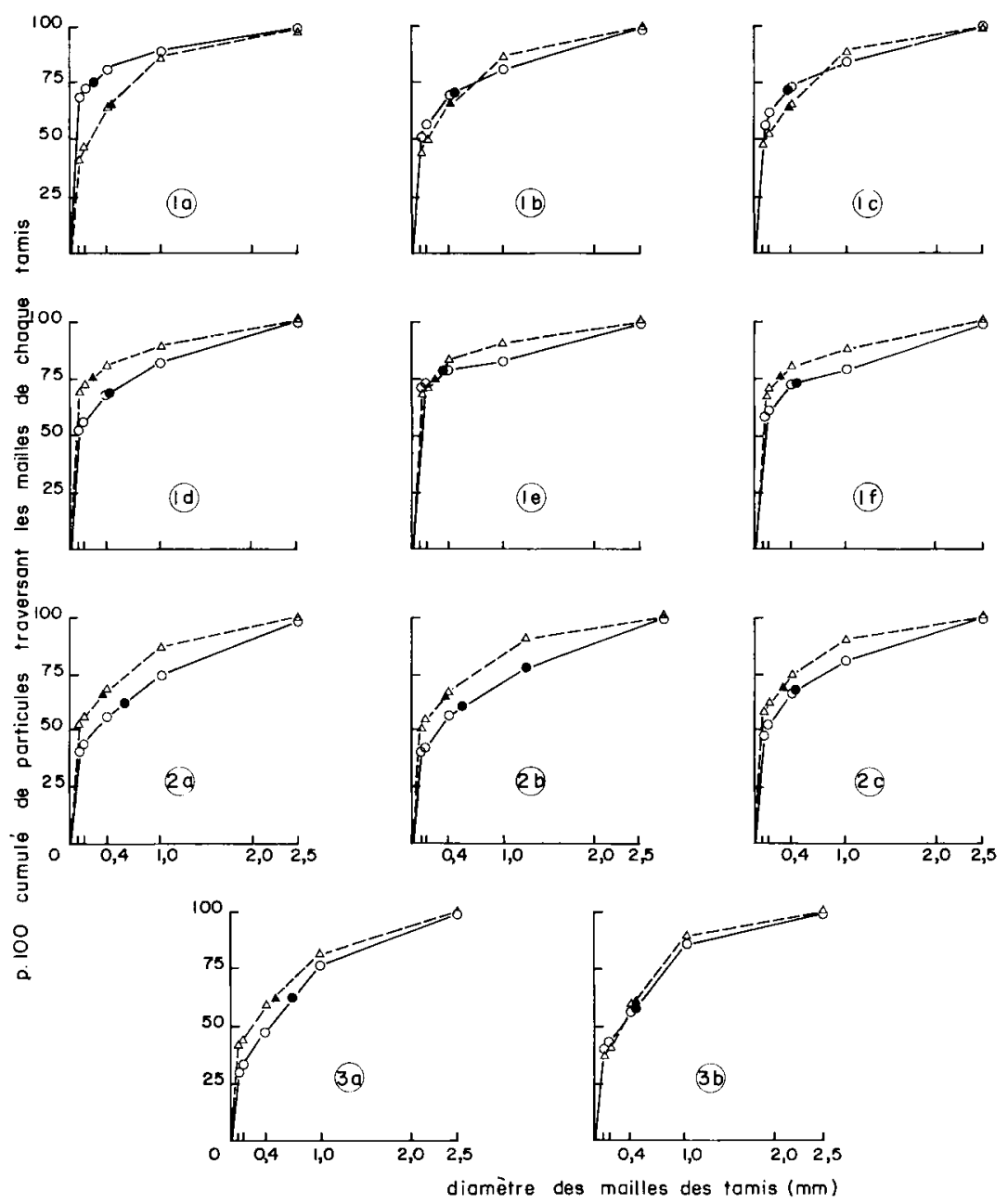

aux tissus cellulosiques diffërenciés par la double coloration, selon la méthode exposée précédemment (GRENET, I966). Une note moyenne par échantilion de toutes les particules de taille supéricure à $0,40 \mathrm{~mm}$ est ensuite calculée.

La proportion des particules issues des aliments concentrés et du maïs a été notée dans les échantillons de contenu de feuillet et de fèces des animaux ayant reçu les régimes $6 a$ et $7 b$, trois animaux par régime étant utilisés pour cette étude et, d'autre part, la recherche des grains d'amidon de mais et d'orge a été effectuée.

\section{RÉSULTATS}

Taille comparée des particules du contemu de feuillet et des fèces

Nous avons représenté les 20 régimes (fig. I) en portant sur chaque graphique la taille des particules du contenu de feuillet et des fèces correspondantes. Ces particules sont de petite taille : la proportion de matière sèche recueillie sur le tamis 

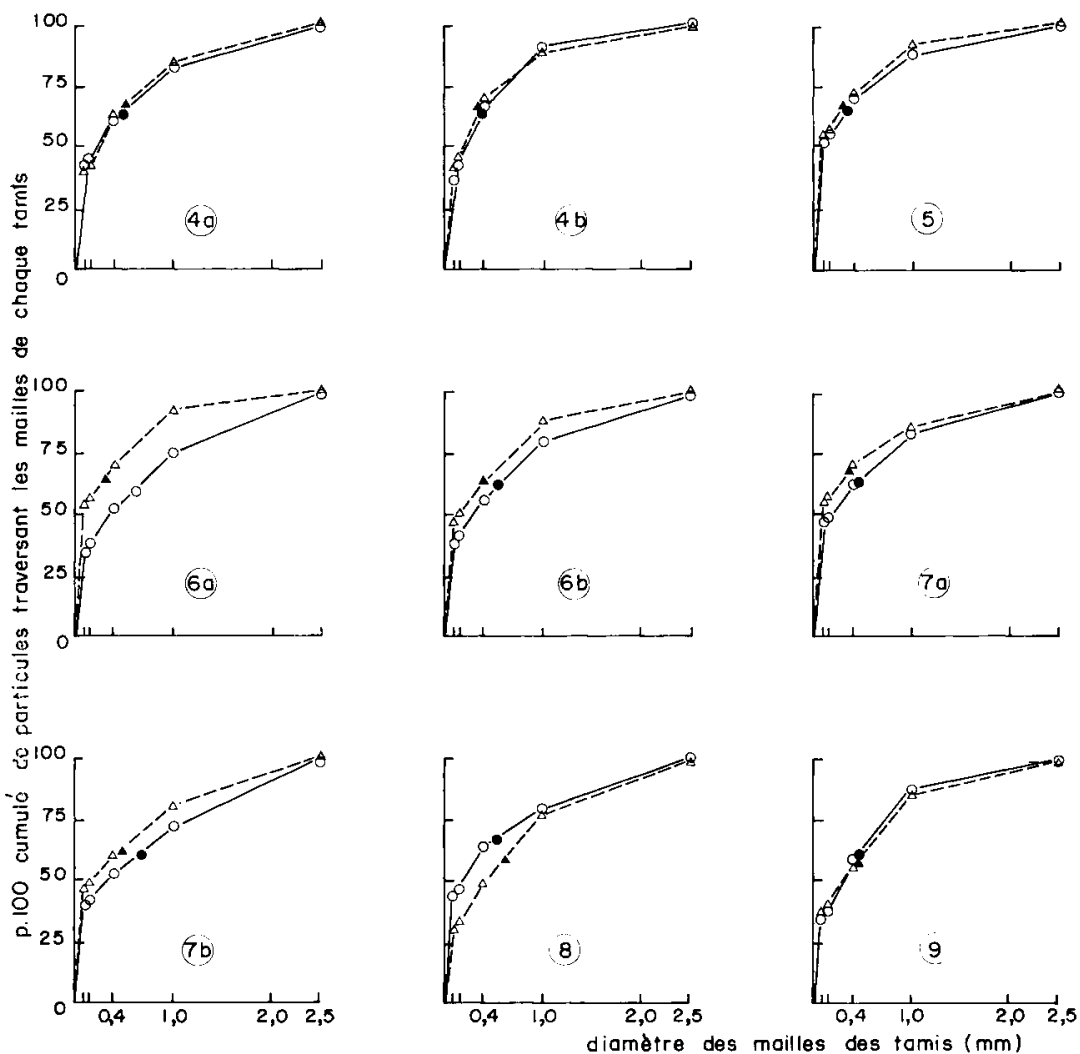

FIG. I. - Pourcentage cumulé des particules du contenu de fenillet et des fèces traversant les mailles de chaque tamis en fonction du diametre des mailles de ces tamis. Taille moyenne des particules

- Contenu de feuillet -..... - Tièces
- Taille moyenne des particules du contenu de feuillet

- Taille moyenne des particules fécales

à mailles de I mm varie de 9 à $28 \mathrm{p}$. Ioo pour le contenu de feuillet, de 8 à $23 \mathrm{p}$. Ioo pour les fèces, tandis que la proportion de matière sèche traversant le tamis de o, Io $\mathrm{mm}$ s'échelonne entre 2 I et $65 \mathrm{p}$. roo pour le contenu de feuillet, 22 à $63 \mathrm{p}$. Ioo pour les fèces.

La taille moyenne des particules du contenu de fetrillet varie de 0,26 à $0,70 \mathrm{~mm}$ suivant les régimes (fig. I). Certaines particules peuvent cependant atteindre des longueurs de $5 \mathrm{~cm}$, mais elles sont présentes en proportion infime. Les échantillons provenant d'animaux ayant pâturé une prairie naturelle ou du ray-grass anglais montrent la présence de très nombreuses particules végétales fines, très souples, emmêlées et longues parfois de ro $\mathrm{cm}$, qui sont plus nombreuses dans le contenu de feuillet que dans les fèces. On peut supposer que ces particules ont franchi l'orifice réticulo-omasal longitudinalement. Par ailleurs, les particules de contenu de feuillet avec des régimes comportant du foin de graminées, ont une forme nettement plus allongée que celles provenant de foins de légumineuses, ces dernières étant plus isodiamétriques. 
Afin de comparer la taille des particules du contenu de feuillet et des particules alimentaires lorsque des foins broyés sont distribués, nous avons mesuré la taille moyenne des particules des deux foins condensés de luzerne et de graminées, nos $3 b$ et $4 b$ : elle est de 0,47 et $0,30 \mathrm{~mm}$, alors que la taille moyenne des particules du contenu de feuillet correspondant est égale ou supérieure : 0,46 et $0,39 \mathrm{~mm}$.

Les particules fécales sont de plus petite taille que les particules du feuillet : suivant les régimes, leur taille moyenne varie de 0,24 à $0,63 \mathrm{~mm}$. La taille des particules fécales de fétuque, fléole et ray-grass d'Italie (nos $1 a, b, c$ ) a été légèrement surestimée par suite d'une agglomération des particules entre elles, difficile à détruire au cours de la séparation des particules selon leur taille, les échantillons de fèces ayant été séchés à l'étuve avant leur étude. $\mathrm{L}_{\text {a }}$ taille moyenne des particules fécales pour les 20 régimes $(0,39 \mathrm{~mm})$ est significativement $(\mathrm{P} \leqslant 0,05)$ inférieure à la taille moyenne des particules du contenu de feuillet $(0,50 \mathrm{~mm})$. I a répartition moyenne des particules du feuillet et des fèces sur les différents tamis (fig. 2) fait apparaître que la proportion de particules de taille supérieure à $\mathrm{I} \mathrm{mm}$ est significativement ( $\mathrm{P} \leqslant 0$,OI) plus grande dans le contenu de feuillet que dans les fèces, les pourcentages de particules recueillies sur les autres tamis n'étant pas significativement différents entre le feuillet et les fèces.

Au-delà de $0,40 \mathrm{~mm}$, et en deçà de 0, Io mm, la distribution des particules n'est pas homogène d'un régime à l'autre (fig. 2) ; les coefficients de variation de la proportion de particules recueillie sur les tamis de I mm, o,40 mm, o, 6 mm, o, ro mm et dans le fond du tamis sont respectivement de 29,8 p. IOO, 4I,3 p. IO0, 29,2 p. IOO, $25,2 \mathrm{p}$. Ioo et $35,5 \mathrm{p}$. IoO pour les contenus de feuillet, et 29,4 p. IOO, 3I,5 p. IOO, $2 \mathrm{I}, 0$ p. IOO, 24,5 p. IOO et $30,7 \mathrm{p}$. IOO pour les fèces.

Il existe une relation très étroite entre la taille moyenne des particules et la quantité de particules recueillie sur les deux premiers tamis (I et $0,40 \mathrm{~mm}$ ), $r=0,9 I I$ et 0,955 respectivement pour les contenus de feuillet et les fèces ; ainsi la mesure de la proportion de particules de taille supérieure à $0,40 \mathrm{~mm}$ serait suffisante pour obtenir une bonne estimation de la taille moyenne des particules.

\section{Relations entre \\ la taille des particules et la valeur alimentaire des fourrages}

Chaque régime comprenait en moyenne $50 \mathrm{p}$. Ioo de fourrage, et les particules du contenu de feuillet et des fèces en proviennent en majeure partie. Aussi avonsnous relié la taille moyenne des particules à ses caractéristiques (tabl. 2). La taille moyenne des particules du feuillet varie en sens inverse de la digestibilité de la matière organique, et dans le même sens que la teneur en matière organique indigestible et que la teneur en cellulose brute indigestible. La taille moyenne des particules fécales montre seulement une relation positive avec la quantité de matière organique non digestible.

\section{Examen microscopique des particules du contenu de feuillet et fes fèces}

\section{Étude qualitative des particules.}

Seules les particules de taille supérieure à $0,40 \mathrm{~mm}$ ont été examinées ; elles sont composées de tissus lignifiés (vaisseaux et sclérenchyme), de parenchyme et d'épi- 


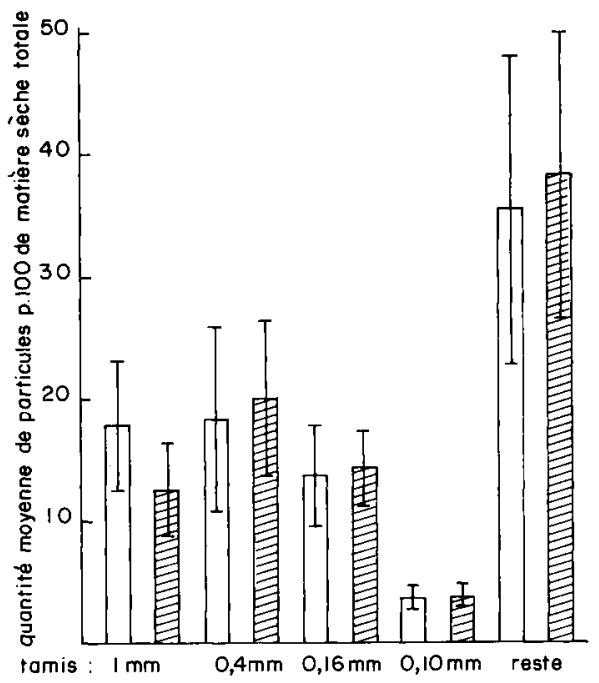

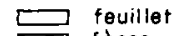 \\ FIG. 2. - Répartition moyenne des particules du contenu du feuillet} et des fèces sur les differents tamis.

\section{TABLEAU 2}

Corrélation entre la taille et la lignification des particules du contenu de feuillet et des fèces et les caractéristiques du fourrage

\begin{tabular}{|c|c|c|c|c|}
\hline \multirow{2}{*}{$\stackrel{x}{\downarrow}$} & \multicolumn{2}{|c|}{ Taille moyenne des particules } & \multicolumn{2}{|c|}{ Lignification } \\
\hline & Feuillet & Fèces & Feuillet & Fèces \\
\hline CUD de la matière organique $(a) \ldots$ & $-0,510^{*}$ & $-0, \geq 81$ & $-0,6 \pm 7 * *$ & $-0,578^{*}$ \\
\hline CUD de la cellulose brute $(a) \ldots .$. & $-0,417$ & $-0,078$ & $-0,678 * *$ & $-0,500^{*}$ \\
\hline Mat. organique non digestible $(a) \ldots$ & $+\cdot 0,5 \geq 6^{*}$ & $+0,486 *$ & $+0,619 * *$ & $+0,601 *$ \\
\hline Cellulose brute non digestible $(a) \ldots$ & $+0,604 *$ & $+0,185$ & $+0,773 *$ & $+0,535^{*}$ \\
\hline Quantité de matiere sèche ingérée & & & & \\
\hline$(\mathrm{kg} / \mathrm{j} / 100 \mathrm{~kg}$ de poids vif) $(b) \ldots$ & $-0,366$ & $-0,095$ & $-0,287$ & $-0,26 \mathbf{k}$ \\
\hline
\end{tabular}

(a) $n=17$

(b) $n=18$

* $r$ significatif pour $P \leqslant 0,05$

** $r$ significatif pour $\mathrm{P} \leqslant 0,01$

derme. Tous les échantillons de contenu de feuillet et de fèces, provenant de foin de graminées ou de foin de luzerne de premier cycle, renferment des cuticules d'épiderme, alors que l'examen de fèces de moutons, alimentés avec du fourrage vert de premier cycle, avait montré que seules les cuticules des épidermes de graminées étaient indigestibles (GRENET, I966). Dans les échantillons correspondant à du foin 
de luzernes nous avons noté la présence de plages de fibre, péricycliques de la tige renfermant des cristaux d'oxalate de calcium et de débris de parenchyme foliaire encadrant des nervures. Nous n'avons pas observé de fragments d'épiderme dans les échantillons de contenu de feuillet et de fèces provenant de vaches alimentées avec du foin de trèfle, mais des plages de tissu cellulosique à petites cellules isodiamétriques. La présence de houlque laineuse dans la prairie naturelle se matérialise dans les contenus de feuillet et les fèces par l'existence de très nombreux poils.

L'examen des contenus de feuillet et des fèces permet d'identifier en grande partie les divers constituants du régime, betteraves et aliments concentrés (FERRANDO et HENRY, r966). Ainsi, on retrouve des fragments du parenchyme de réserve des betteraves, de 1'assise scléreuse du tourteau de colza, de l'épiderme, de l'assise protéique et du parenchyme des grains de céréales (maïs, orge, avoine).

Le tableau 3 indique la proportion de particules issues de l'aliment concentré par rapport at fourrage dans les contenus de feuillet et les fèces pour les régimes $\mathrm{n}^{\mathrm{os}} 6 a$ (50 p. Ioo de foin et $50 \mathrm{p}$. Ioo de maïs) et $7 b(25 \mathrm{p}$. Ioo de foin et $75 \mathrm{p}$. Ioo de concentré) : elle est plus importante dans les fèces que dans le contenu de feuillet; pour le régime $n^{\circ} 6 a$, la proportion de fragments de parenchyme diminue dans les fèces, faisant apparaître la possibilité d'une digestion des cellules parenchymateuses au niveau intestinal.

La recherche de l'amidon de maïs et d'orge a été effectuée dans les échantillons de contenu de feuillet et de fèces de ces deux régimes nos $6 a$ et $7 b$. Le maïs était cru, sous forme de mouture grossière, et l'orge était broyée à la grille de $4 \mathrm{~mm}$ puis

TABLEAU 3

Proportion de particules issues de l'aliment concentré dans les contenus de feuillet et les fèces correspondant aux régimes $6 \mathrm{a}$ et $7 \mathrm{~b}$

\begin{tabular}{|c|c|c|c|c|c|c|c|c|}
\hline & \multicolumn{4}{|c|}{$\begin{array}{c}6 a: \text { foin de luzerne }+5 \mathrm{~kg} \text { de maiss } \\
\text { (mouture grossière) }\end{array}$} & \multicolumn{4}{|c|}{$\begin{array}{c}7 b: \text { foin de trèfle }+ \text { ensilge } \\
+7 \mathrm{~kg} \text { de concentré (1) }\end{array}$} \\
\hline & \multicolumn{2}{|c|}{ Feuillet } & \multicolumn{2}{|c|}{ Feces } & \multicolumn{2}{|c|}{ Feuillet } & \multicolumn{2}{|c|}{ Fèces } \\
\hline & $1 \mathrm{~mm}$ & $0,1 \mathrm{~mm}$ & $1 \mathrm{~mm}$ & $0,4 \mathrm{~mm}$ & $1 \mathrm{~mm}$ & $0,4 \mathrm{~mm}$ & $1 \mathrm{~mm}$ & $0,{ }^{\prime} \mathrm{mm}$ \\
\hline $\begin{array}{l}\text { Particules d'aliment concentré } \\
\qquad \%) \text { (500 partic. examinées) }\end{array}$ & 7 & 7 & 12 & 15 & 30 & 23 & 30 & 46 \\
\hline $\begin{array}{l}\text { P. } 100 \text { particules } \\
\text { d'aliment concentré }\end{array}$ & & & & & & | & & \\
\hline $\begin{array}{l}\text { Épiderme (céréales) ......... } \\
\text { Parenchyme (céréales) ...... } \\
\text { Tourteau de colza ........ } \\
\text { Bois d'origine indéterminée. }\end{array}$ & $\begin{array}{l}27 \\
73\end{array}$ & $\begin{array}{l}15 \\
55\end{array}$ & $\begin{array}{l}7 / \\
26\end{array}$ & $\begin{array}{l}84 \\
16\end{array}$ & $\begin{array}{r}58 \\
6 \\
10 \\
12\end{array}$ & $\begin{array}{r}52 \\
9 \\
19 \\
3\end{array}$ & $\begin{array}{r}63 \\
8 \\
9 \\
13\end{array}$ & $\begin{array}{r}51 \\
7 \\
19 \\
7\end{array}$ \\
\hline
\end{tabular}

(1) Concentré composé de 60 p. 100 d'orge, 15 p. 100 d'avoine, 10 p. 100 de tourteau d'arachide et 10 p. 100 de tourteau de colza. 
agglomérée ; $3 \mathrm{~g}$ environ de contenu de feuillet ou de fèces ont été mis en suspension dans un cristallisoir avec $60 \mathrm{ml}$ d'eau, puis une goutte de la suspension était placée entre lame et lamelle et additionnée de 2 gouttes de $\mathrm{Lugol}\left(\mathrm{I}_{2}+\mathrm{IK}\right)$ qui fait apparaître les grains d'amidon noir bleuté. Cetux de maïs sont polygonaux avec un hile punctiforme ou étoilé tandis que les grains d'amidon d'orge sont discoïdes avec un hile allongé. Deux préparations par animal ont été examinées et le nombre de grains d'amidon compté. En moyenne 45I grains d'amidon ont été observés dans les préparations de contenu de feuillet provenant du régime à base de mais, contre 96 dans les fèces. Par contre, 84 grains d'amidon seulement ont été trouvés dans les préparations de contenu de feuillet ainsi que dans les préparations de fèces provenant du régime à base d'orge. Ces résultats sont en accord avec les travaux des chercheurs anglais et américains (Armstrong, SEeleiy et MCRae, I967; Lit'TtLe, Mitcheir, et Reitnour, Ig68) et avec ceux de Thivend et Journerr (I968) à la Station sur la digestion de l'amidon dans le tube digestif ; ces auteurs ont montré qu'une quantité variable d'amidon de mais, pouvant atteindte $30 \mathrm{p}$. Ioo de la quantité ingérée, échappait à l'amylolyse dans le rumen, tandis que l'amidon d'orge y était dégradé à 95 p. Ioo environ. La proportion de grains corrodés par rapport au nombre total de grains d'amidon est de $62 \mathrm{p}$. Ioo en moyenne dans le contenu de fetillet, et de 67 p. Ioo dans les fèces, pour les deux régimes considérés, les variations observées entre les animaux étant plus importantes que les différences entre régimes.

\section{Lignification des particules du contenu de feuillet et des fèces.}

Nous avons évalué la proportion de tissus lignifiés dans les particules de taille supérieure à $0,40 \mathrm{~mm}$ par une note allant de 0 à IO. La note varie de 2,6 à 7 pour les contenus de feuillet, et de 3,5 à 5,9 pour les fèces (tabl. I) ; cette différence n'est pas significative.

En multipliant cette note de lignification par la quantité de particules de taille supérieure à $0,40 \mathrm{~mm} \mathrm{p}$. Ioo de la matière sèche, nous avons obtenu une estimation de la lignification du contenu de fetuillet et des fèces; il existe une liaison positive entre elles : $r=+0,425(\mathrm{P} \leqslant 0,05)$. La lignification des particules du feuillet et des fèces présente une liaison négative avec la digestibilité de la matière organique et de la cellulose brute des fourrages, et une liaison positive avec la quantité de matière organique indigestible et de cellulose brute indigestible (tabl. 2). Ces résultats sont semblables à ceux que nous avions obtenus sur moutons.

\section{DISCUSSION}

Les résultats que nous avons obtenus confirment la nécessité d'une réduction des aliments à l'état de particules extrêmement fines, de l'ordre du mm, pour franchir l'orifice du feuillet chez les bovins. Les particules longues de plusieurs cm, mentionnées plus haut, étaient peu nombreuses, et on peut supposer qu'elles avaient franchi l'orifice du feuillet longitudinalement. Cependant, des différences de taille entre les particules apparaissent suivant l'espèce fourragère ingérée, ces différences étant dues à la forme plus ou moins allongée des particules; l'étude microscopique des plantes fourragères devrait permettre d'expliquer cette différence. Ceci rejoint les résultats 
obtenus à la même époque par Troessen et CAMPBELL (I968), sur 5 espèces fourragères différentes. Ces auteurs ont relié les variations de la taille des particules au comportement alimentaire des animaux, particulièrement à la quantité de matière sèche ingérée : nous n'avons pas pu mettre de liaison en évidence entre la taille des particules et la quantité de matière sèche ingérée, essentiellement à cause de l'hétérogénéité et de la complexité des régimes.

Les particules du contenu de feuillet sont de taille supérieure aux particules du foin condensé ingéré. Le fait que les animaux consomment la paille de leur litière lorsqu'ils reçoivent des foins condensés, peut expliquer cette augmentation de taille. D'autre part, les plus fines particules de foin, de nature cytoplasmique, doivent être entièrement dégradées dans le rumen, ce qui a pour effet d'accroître la proportion des grandes particules, et par suite d'élever la taille moyenne. Néanmoins, ces résultats sont à rapprocher de ceux obtenus par DEMARquirLy et JoURnET (I967) sur la vitesse de transit accélérée des foins condensés distribués ad libitum, les particules alimentaires étant d'une finesse telle qu'elles peuvent franchir l'orifice du feuillet sans réduction préalable.

Les particules fécales sont de taille un peu moindre que les particules de content1 de feuillet : ceci rejoint les résultats obtenus par PEARCE (I967) sur moutons. Cette diminution de taille est probablement le résultat des pressions mécaniques et des actions enzymatiques exercées le long du tube digestif. Les études effectuées sur la digestion intestinale de la cellulose (cf. revues de ARMstrong et BEEvER, I969 et JouRNET, résultats non publiés) montrent qu'elle est de Io p. Ioo environ pour des moutons ou des bovins consommant du fourrage vert, du fourrage déshydraté ou du foin ; pour des moutons recevant de la paille de blé, HoGAN et WESTON (I967) ont trouvé qu'elle s'élevait à 30 p. roo; Thоmson et al. (I969), sur des moutons alimentés avec de la luzerne déshydratée condensée, ont mesuré une digestion intestinale de la cellulose encore plus importante $(36,7 \mathrm{p}$. I0o). Avec des régimes de fourrages et de céréales, les valeurs obtenues par MiтchELL et al. (I967) sur bovins varient de o à $23,8 \mathrm{p}$. Ioo, les plus élevées correspondant aux régimes les plus riches en céréales. Le lieu de cette digestion, étudiée sur des moutons et des bovins alimentés avec des fourrages ou des régimes mixtes par BRUCE et al. (I966), MCRAE et Armstrong (I969), Thomson et al. (I969) et Journet (résultats non publiés), est généralement le cæcum et le côlon, cependant une partie de la digestion peut avoir lieu dans l'intestin grêle, comme c'est le cas pour le régime de luzerne déshydratée condensée cité plus haut, ou pour les régimes mixtes très riches en céréales. La concentration en acides gras volatils dans le cæcum et le côlon montre l'importance de la fermentation microbienne à ce niveau (BOYNE et al., 1956; BADAwy et al., I958). Une proportion variable des hémicelluloses digestibles (Io à 30 p. Ioo) disparaît également dans le cæcum et le côlon (ARMSTRong et BEEvER, I969; McRAE et Armstrong, Ig69).

Les particules fécales de moutons (GRENET, I966) sont de taille légèrement inférieure aux particules fécales de bovins : respectivement 0,1 à $0,3 \mathrm{~mm}$ et 0,2 à $0,6 \mathrm{~mm}$. Cependant, cet écart est de faible importance, eu égard à la différence de format entre les deux espèces. Il semble qu'il y aurait peu de relation entre la taille des particules et le volume des réservoirs gastriques, la réduction en fines particules étant principalement le fait des micro-organismes du rumen. 
Les liaisons que nous avons obtenues entre la taille des particules du contenu de feuillet et des fèces de bovins et la digestibilité des fourrages ingérés sont moins étroites que celles que nous avions trouvées lors de l'étude des fèces de moutons. Cette différence s'explique par le fait que nous avons affaire cette fois-ci non pas à des fourrages verts, mais à des régimes complexes et avec des proportions variables d'aliments concentrés.

Les épidermes de foin de luzerne de premier cycle apparaissent ici indigestibles, contrairement aux épidermes de luzerne de premier cycle distribuée en vert lors de l'étude des fèces de moutons. Ceci est à mettre en parallèle avec les modifications physico-chimiques et la perte de digestibilité qui accompagnent la dessiccation d'un fourrage : en effet, les teneurs en azote et en glucides solubles d'un fourrage de luzerne ou de graminées diminuent lors du séchage (RAGuss et SMITH, I965; RHYKERD et al., I966; NOLLER et al., I966), et les digestibilités in vitro de la matière sèche et de la cellulose de la luzerne séchée à une température de $60^{\circ} \mathrm{C}$ subissent une baisse sensible, respectivement de 3,8 et 2,4 points (NoLlER et al., I966).

D'autre part, l'examen microscopique des contenus digestifs permet de déterminer les différents composants de l'aliment concentré de la ration, la nature de l'amidon et le lieu de sa digestion, et peut apporter une confirmation aux résultats des analyses chimiques.

L'étude de la taille des particules du contenu de feuillet et des fèces de bovins, et leur observation microscopique permet de conclure que, par l'examen des fèces des ruminants, on obtient une bonne image de la digestion effectuée dans le rumen.

Reçu pour publication en septembre 1970.

\section{SUMMARY}

SIZE AND STRUCTURE OF PLANT PARTICLES IN THE

OMASUM AND FAECES OF CATTLE

I. Fifty samples of contents of the omasum and 48 samples of faeces were studied; they were from 50 young bulls, bullocks and cows which had been given 20 different diets. Of the feeds 6 were of green fodder and the other 14 were winter fattening rations based on forage and concentrates in different proportions (table r). The size of the particles was measured, and the nature of the plant particles and the proportion of lignified membranes which they contained were studied microscopically.

2. The distribution of particles from the omasum and faeces on the 4 screens with respective mesh openings of $\mathrm{I} \mathrm{mm}, 0.40 \mathrm{~mm}, 0.16 \mathrm{~mm}$ and $0.10 \mathrm{~mm}$, and in the tray is shown in figure I. The average size of particles in the omasum was small, ranging from 0.26 to $0.70 \mathrm{~mm}$, depending on the diet. There were, however, some particles several centimetres long which had probably slipped lengthwise through the orifice from the rumen. Also there were differences in size of particles among species, since legumes gave rise to particles of more equal diameter than did grasses. Particles in faeces were a little smaller than those in the omasum, from 0.24 to $0.63 \mathrm{~mm}$. The average distribution of particles from omasum and faeces on different screens (fig. 2) shows that the proportion of particles greater than I $\mathrm{mm}$ was significantly greater in omasum contents than in faeces. The average size of particles in the omasum varied inversely with the digestibility of organic matter of the forage and directly with the contents of indigestible organic matter and indigestible crude fibre (table 2). The average size of particles in faeces was not significantly related to digestibility of the forage and showed a positive relation only with indigestible organic matter. 
3. Particles in omasum contents and in faeces larger than $0.4^{\circ} \mathrm{mm}$ were composed of lignified tissue (vessels and sclerenchyma), parenchyma and epidermis. Presence of epidermal cuticle in samples deriving from grass hay or lucerne hay of the first cycle was general. Examination of contents of omasum and faeces allowed to a large extent the identification of the constituents of the diet, beet and concentrates (table 3) and the estimation of the site of digestion of the starch of cereals.

4. Lignification in particles of the omasum contents was positively related to lignification of particles in faeces $(r=+0.425)$. Both were negatively related to digestibility of organic matter and of crude fibre of the forage and positively to contents of indigestible organic matter and indigestible crude fibre (table 2 ).

5. The results are discussed, particularly the smaller size of particles in faeces in comparison to those in the omasum; this is probably the result of pressure and of enzymic activity in the digestive tract.

On the other hand, the reduced and similar size of particles in faeces of cattle and sheep shows that there would not be a close relation between particle size and the size of the species and consequently with the volume of the gastric reservoirs. This is explained by the fact that the reduction to very fine particles is mainly due to the micro-organisms in the rumen.

\section{RÉFÉRENCES BIBLIOGRAPHIQUES}

Armstrong D. G., Beever D. E., ig69. Post-abomasal digestion of carbohydrate in the adult ruminant. Proc. Nutr. Soc., 28, I2I-I3I.

Armstrong D. G., Seeley R. C., Mac Rae J. C., ig67. Feed carbohydrates. The contribution of the end-products of their digestion to energy supply in the ruminant. IV. Symposium sur le métabolisme ínergétique. Varsovie.

Badawy A. M., Campbell R. M., Cuthbertson D. P., Mackie W. S., I958. Further studies on the changing composition of the digesta along the alimentary tract of the sheep. II. Volatile fatty acids and energy relative to lignin. Br. J. Nutr., 12, 384-39o.

Becker R. B., Marshall S. P., Dix Arnold P. T., 1963. Anatomy, development and functions of the bovine omasum. J. Dairy Sci., 46, 835-839.

Boyne A. W., Campbell R. M., Davidson J., Cuthibrtson D. P., I956. Changes in composition of the digesta along the alimentary tract of sheep. Br.J. Nutr., 10, 325-333.

Brlce J., Goodall E. D., Kay R. N. B., Pinllipson A. T., Vowles L. E., ig66. The flow of organic and inorganic materials through the alimentary tract of the sheep. Proc. Royal Soc., série $B$, 166, $46-63$.

Demarquilly C., Jolrnet M., I967. Valeur alimentaire des foins condensés. I. Influence de la nature du foin et de la finesse de broyage sur la digestibilité et la quantité ingérée. Ann. Zootech., 16, I23-I50.

Ferrando R., Henry N. Détermination microscopique des composants des aliments du bétail. Vigot. Frères. Paris, 1966.

Grenet E., 1966. Les particules végétales des fèces de mouton. Ann. Zootech., 15, 303-3I2.

Hogan J. P., WEston R. HI., I967. The digestion of chopped and ground roughages by sheep. II. The digestion of nitrogen and some carbohydrate fractions in the stomach and intestines. Aust. J. A gric. Res., 18, 803-819.

Journet $M$. Résultats non publiés.

Little C. O., Mitchell. G. E., Reitnour C. M., ig68. Post-ruminal digestion of corn starch in steers. J. Anim. Sci., 27, 790-792.

Mac Rae J. C., Armstrong D, G., ig69. Studies on intestinal digestion in the sheep. II. Digestion of some carbohydrate constituants in hay, cereal and hay-cereal rations. Br. J. Nutr., 23, 377-387.

Mitchell G. F. Jr., Little C. O., Karr M. R., Hayes B. W., I967. Ruminal and post-ruminal digestion of cellulose in steers. $J$, Anim. Sci., 26, 224, (Abst.).

Noller C. H., Prestes P. J., Rhykerd C. I., Rumsey T. S., Burns J. C., ig66. Changes in chemical composition and digestibility of forages with method of sample handling and drying. Proc. 10th Internation.. Grassl. Congr., I966. Helsinki.

Pearce G. R., r967. Changes in particule size in the reticulo-rumen of sheep. Aust. J. Agric. Res., 18, IIg-I25.

Raguse C. A., Smith D., I965. Carbohydrate content in alfalfa herbage as influenced by methods of drying. J. Agric. Food. Chem., 13, 306-309.

Rhykerd C. L., Dillon J. E., Noller C. H., Burns J. C., ig66. The influence of nitrogen fertilization and drying method on yield and chemical composition of Dactylis glomerata, Bromus inermis and Phleum pratense. Proc. 10th internat. Grassl. Congr., Ig66. Helsinkj. 
Smith L. W., Waldo D. R.. Moore L. A., Leffel E. C., Van Soest P. J., r967. Passage of cell wall constituents in the sheep. Paper P. Ioy ann. meet. Amer. Dairy Sci Ass., Ithaca, New-York.

Thivend P., Journet M., x968. Utilisation digestive de l'amidon de l'orge chez le ruminant, $A n n$. Biol. anim. Bioch. Biophys., 8, 449-451.

Thomson D. J., Benver D. F., Coehlo da Silva J. F., Armstrong D. G., I969. Sites of digestion in sheep of a dried lucerne fed in three different physical forms. Proc. Nutr. Soc., 28, 24 A.

Troelsen J. E., CAmpbell J. B., I968. Voluntary consumption of forage by sheep and its relation to the size and shape of particles in the digestive tract. Anim. Prod., 10, 289-296. 